\title{
AVALIAÇÃO DA CONTAMINAÇÃO POR PESTICIDAS NOS SEDIMENTO DO RIO FORMOSO NO ESTADO DO TOCANTINS
}

\author{
Evaluation of contamination by pesticides in the sediments of Formoso River in the \\ state of Tocantins
}

Evaluación de la contaminación por pesticidas en los sedimentos del río Formoso en el estado de Tocantins.

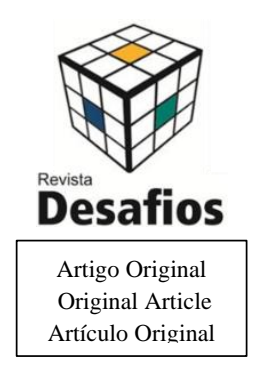

Patricia Martins Guarda" ${ }^{* 1}$, Antonina M. S. Pontes ${ }^{1}$, Raquel de S. Domiciano ${ }^{1}$, Larissa da S. Gualberto $^{1}$, Emerson Adriano Guarda ${ }^{1}$

${ }^{1}$ Laboratório de Pesquisa em Química Ambiental e Biocombustíveis, Universidade Federal do Tocantins, Palmas, Brasil.

*Correspondência: Laboratório de Pesquisa em Química Ambiental e Biocombustíveis, Instituto de Ensino Superior, Av. NS 15, 109 Norte, Palmas, Tocantins, Brasil.CEP:77.010-090.e-mail patriciaguarda@uft.edu.br.

\section{RESUMO}

Considerando o aumento da produção agrícola do Tocantins e o aumento no uso de pesticidas para esta produção, foram investigados os níveis de resíduos de pesticidas em amostras de sedimento do Rio Formoso, no estado do Tocantins. Analisou-se a presença de três pesticidas da classe dos carbamatos (carbaril, carbofurano e propoxur) por UHPLC-MS/MS, nos períodos de abril/2018 e julho/2019. As coletas foram realizadas as margens deste rio, na área de influência do projeto agrícola de Formoso do Araguaia TO. Não foram encontrados os princípios ativos nas amostras de sedimento, o que pode ser entendido em função de algumas características físico químicas dos pesticidas estudados. Utilizando o método GOSS, os pesticidas estudados apresentaram características de rápida degradação no sedimento e com baixo potencial de contaminação associado ao sedimento. Foram analisadas, também, as características físico-químicas das amostras de sedimento coletadas, que justificam essa não quantificação desses três princípios ativos, nesse compartimento ambiental. Ainda que nenhum dos princípios ativos tenham sido encontrados, não se pode dizer que estes não estejam presentes em outros compartimentos, já que apresentam alto potencial de contaminação de água subterrânea, pelo método GUS e EPA.

Palavras-chave: Carbamatos, sedimento, método de GOSS.

\section{ABSTRACT}

Considering the increase in agricultural production of Tocantins and the increase in the use of pesticides for this production, pesticide residue levels were investigated in sediment samples from the Formoso River in the state of Tocantins. The presence of three pesticides of the carbamate class (carbaryl, carbofuran and propoxur) was analyzed by UHPLC-MS/MS, in the periods of April/2018 and July/2019. The banks of this river were collected in the area of influence of the agricultural project of Formoso do Araguaia - TO. The active ingredients in sediment samples were not found, which can be understood due to some chemical physical characteristics of the pesticides studied. Using the GOSS method, the pesticides studied presented characteristics of rapid sediment degradation and with low contamination potential associated with sediment. The physicochemical characteristics of the sediment samples collected were also analyzed, which justify this non-quantification of these three active ingredients in this environmental compartment. Although none of the active ingredients have been found, it cannot be said that they are 
not present in other compartments, since they have a high potential for groundwater contamination, by the GUS and EPA method.

Keywords: Carbamates, sediment, GOSS method.

\section{RESUMEN}

Teniendo en cuenta el aumento de la producción agrícola de Tocantins y el aumento en el uso de pesticidas para esta producción, se investigaron los niveles de residuos de plaguicidas en muestras de sedimentos del río Formoso en el estado de Tocantins. La presencia de tres plaguicidas de la clase carbamato (carbarilo, carbofurano y propoxur) fue analizada por UHPLC-MS/MS, en los períodos de abril/2018 y julio/2019. Las orillas de este río fueron recogidas en la zona de influencia del proyecto agrícola de Formoso do Araguaia - TO. No se encontraron los principios activos de las muestras de sedimentos, que pueden entenderse debido a algunas características físicas químicas de los plaguicidas estudiados. Utilizando el método GOSS, los pesticidas estudiados presentaron características de degradación rápida de sedimentos y con bajo potencial de contaminación asociado con sedimentos. También se analizaron las características fisicoquímicas de las muestras de sedimentorecogidas, que justifican esta no cuantificación de estos tres ingredientes activos en este compartimento ambiental. Aunque no se ha encontrado ninguno de los ingredientes activos, no se puede decir que no estén presentes en otros compartimentos, ya que tienen un alto potencial de contaminación de las aguas subterráneas, por el método GUS y EPA.

Descriptores: Carbamatos, sedimento, método de GOSS.

\section{INTRODUÇÃO}

O mercado brasileiro de agrotóxicos expandiu rapidamente na última década, em um ritmo de crescimento maior que o dobro do apresentado pelo mercado global, o que coloca o Brasil em primeiro lugar no ranking mundial, desde 2008 (ANVISA, 2013).

A intensificação do uso de agrotóxicos e a não utilização de práticas conservacionistas comprometem a qualidade do solo e das águas superficiais e subterrâneas, acarretando sérios impactos ao meio ambiente e a saúde humana (CALDAS e SOUZA, 2000).

Com a expansão agrícola, temos o aumento do consumo de agrotóxicos, os quais constituem insumos de fundamental importância no manejo de pragas, plantas daninhas e agentes causadores de doenças, todavia, o uso inadequado desses produtos pode provocar efeitos indesejáveis nos mais diferentes compartimentos ambientais (ADAPEC, 2008).

O território do Estado está em posição geográfica estratégica e aliado às condições ambientais favoráveis, clima, recursos hídricos, solo e vegetação, torna-se o espaço ideal para a expansão da agricultura. $\mathrm{O}$ estado tem na agropecuária a sua principal fonte econômica, e a partir da década de 1990 tem intensificado a área desmatada de cerrado para o cultivo da monocultura (SOARES et al., 2013). Os produtos que mais se destacaram na região estudada são: soja para semente, melancia e feijão no período de seca e, cana-de açúcar e arroz no período chuvoso.

A média anual do uso de agrotóxico no Tocantins é de 9.715 toneladas/ano, e a relação entre a média anual do uso de agrotóxico em $\mathrm{kg}$ e a área agrícola em hectare fica de 4,27 a 7,55 Kg/há (BOMBARDI, 2019).

Os impactos ambientais sobre o solo, água e sua microbiota causados pelo uso dos agrotóxicos estão relacionados principalmente com o tempo de permanência de seus resíduos, nesses compartimentos acima do necessário para exercer sua ação (RIBEIRO e VIEIRA, 2010). O destino dos agrotóxicos no ambiente é governado por processos de retenção, de transformação e de transporte, e por interações entre 
esses processos (SPADOTTO, 2006). Alguns tipos de agrotóxicos, ao permanecerem no ambiente ou atingirem o meio aquático, oferecem riscos para espécies animais por sua toxicidade e possibilita a bioacumulação ao longo da cadeia alimentar (MILHOME, 2009).

A lixiviação e o escoamento superficial podem ocorrer quando a água da chuva segue dois caminhos diferentes. Quando a água infiltra no solo e percola em profundidade, temos o que chamamos de lixiviação. É desta forma que os agrotóxicos podem atingir as águas subterrâneas. A tendência de um agrotóxico se lixiviar depende da sua capacidade de adsorção ao solo, solubilidade em água e tipo de solo. Esses parâmetros físico químicos são de extrema importância no estudo da capacidade de contaminação de águas subterrâneas. Algumas condições ambientais como solo poroso, pluviosidade anual maior que $250 \mathrm{~mm}$ e aquífero confinado favorecem a percolação (REBELO e CALDAS, 2014).

O escoamento superficial é o processo onde a água não se infiltra no solo, ela escorre até os rios durante a chuva, ou logo após elas acabarem, e desta forma atingem as águas superficiais.

Em ambos os casos, tipo de solo, preservação da cobertura vegetal, mata ciliar, manejo agrícola, declividade do relevo e incidência de chuvas interferem no processo (DELLAMATRICE e MONTEIRO 2014).

Em áreas de atividade agrícola, a principal preocupação é a contaminação dos recursos hídricos com resíduos de agrotóxicos, sendo que o principal mecanismo para avaliar o impacto do setor e promover políticas voltadas à saúde e o meio ambiente é o monitoramento da qualidade das águas (GAMA et al., 2013). Esse monitoramento pode ser realizado através de análises laboratoriais periódicas da água e/ou solo, em função dos agrotóxicos utilizados em uma determinada cultura (POSSAVATZ, 2014; FILIZOLA et al., 2016). Dessa forma, estudos das propriedades físico-químicas, acompanhados do uso de modelos de avaliação de risco são alternativas utilizadas para avaliar os riscos de contaminação em grandes áreas agrícolas no Brasil (GAMA et al., 2013). Uma vez determinado o princípio ativo, é possível através de sua estrutura química, determinar a dinâmica do agrotóxico no ambiente, incluindo sua mobilidade $\mathrm{e}$ degradabilidade (SOARES et al., 2016).

Os resultados desses estudos normalmente são apresentados na forma de índices e intervalos numéricos, tais como os critérios da Agencia de Proteção Ambiental Norte-Americana (EPA, do inglês Environmental Protection Agency), o índice de vulnerabilidade de aguas subterrâneas (GUS) (do inglês Groundwater Ubiquity Score), e o método de GOSS, que são os critérios de avaliação do potencial de contaminação de aguas superficiais (GOSS, 1992; ANDRADE et al., 2011), os pesticidas são classificados em alto e baixo potencial de contaminação em função do transporte associado aos sedimentos ou dissolvidos em água (MARQUES, 2005), todos descritos abaixo.

As propriedades de maior interesse no estudo dos agrotóxicos são: Solubilidade em água, Coeficiente de adsorção $\left(\mathrm{K}_{\mathrm{oc}}\right)$, Coeficiente de partição octanol/água $\left(\mathrm{K}_{\mathrm{ow}}\right)$, Constante da Lei de henry $\left(\mathrm{K}_{\mathrm{H}}\right)$, Pressão de Vapor (PV) e Tempo de meia vida $\left(\mathrm{DT}_{50}\right)^{9,17}$. Essas propriedades influenciam diretamente nos processos de transformação, remoção e transporte descritos no item anterior. Os critérios da EPA envolvem os valores de solubilidade em água a $25^{\circ} \mathrm{C}>30 \mathrm{mg} \mathrm{L}^{-1}$; Koc $<300$ $500 \mathrm{~cm}^{3} \mathrm{~g}^{-1}$; constante da Lei de Henry $(\mathrm{KH})<10^{-2} \mathrm{~Pa} \mathrm{~m}^{3}$ $\mathrm{mol}^{-1}$, e t1/2 em solo 14 a 21 dias e na água >175 dias. Agrotóxicos que se enquadram nessas características são considerados potencialmente poluidores de águas 
subterrâneas (MARTINI et al., 2012; SOARES et al. 2016).

O risco de contaminação de águas subterrâneas pode ser realizado com índice de GUS e de acordo com os critérios da EPA (FERRACINI et al 2001; PRIMEL et al., 2005; SOARES et al., 2016). O índice de GUS considera os valores de meia-vida dos compostos no solo ( $\mathrm{DT}_{50 \text { solo }}$ ) e do $\mathrm{K}_{\mathrm{OC}}$, não levando em consideração outras propriedades físico-químicas (GUSTAFSON, 1985; PRIMEL et al., 2005).

O método GOSS é um modelo de potencial de contaminação usado para prever uma possível contaminação de águas superficiais por pesticidas em relação a aqueles que podem ser transportados através de sua dissolução em água e aqueles transportados para águas superficiais associados aos sedimentos em suspensão no corpo hídrico (MILHOME, 2009).

$\mathrm{Na}$ Tabela 1 está apresentado algumas características físico químicas dos pesticidas estudados neste artigo.

Tabela 1. Propriedades físicas e químicas dos pesticidas estudados

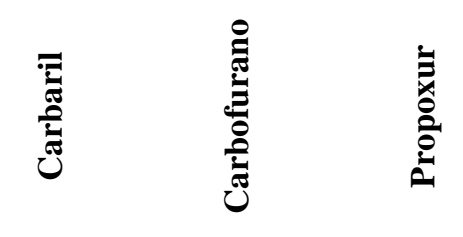

\begin{tabular}{|c|c|c|c|}
\hline$S_{w}\left(m_{g} \cdot L^{-1}\right)$ & 9,1 & 322 & $1,8 \times 10^{3}$ \\
\hline$K_{o c}\left(m L \cdot g^{-1}\right)$ & 300 & 86,5 & 30 \\
\hline DT $_{50}$ solo (dias) & 16 & 29 & 79 \\
\hline $\begin{array}{l}\text { DT }_{50} \text { água } \\
\text { (dias) }\end{array}$ & 12 & 37 & 180 \\
\hline $\begin{array}{c}\text { DT }_{50 \text { sedimento }} \\
\text { (dias) }\end{array}$ & 5,8 & 9,7 & 3,7 \\
\hline $\begin{array}{c}\mathrm{K}_{\mathrm{H}} \\
\left(\text { Pa.m }^{\mathbf{3}} \mathbf{m o l}^{-1}\right)\end{array}$ & $9,2 \times 10^{-5}$ & $5 \times 10^{-5}$ & $1,50 \times 10^{-4}$ \\
\hline $\log K_{\text {ow }}$ & 2,36 & 1,8 & 0,14 \\
\hline PV (mPa) & $4,16 \times 10^{-3}$ & $8 \times 10^{-3}$ & 1,3 \\
\hline
\end{tabular}

Fonte: Adaptado de (KIDD et al., 1991; WAUCHOP et al., 1992; HANSH et al., 1995; SANTOS, 1999; MILHOME et al., 2009; LIMA, 2017; PPDB, 2018;
PUBCHEM, 2018; ARIADNE, 2019).

De acordo com o valor obtido, os princípios ativos são classificados de acordo com a tendência a sofrer lixiviação, conforme os seguintes intervalos: GUS $<1,8$ (não sofre lixiviação); 1,8<GUS<2,8 (faixa de transição); GUS>2,8 (provável lixiviação).

Para águas superficiais, o mais utilizado é o método de GOSS, baseado nos parâmetros de DT50 solo, $\mathrm{Sol}_{\mathrm{w}}$ e Koc, avalia o potencial do agrotóxico de ser transportado dissolvido em água ou associado ao sedimento em suspensão (MILHOME, 2009; MARTINI et al., 2012; SOARES et al., 2016).

Em relação à toxidade os agrotóxicos podem ser classificados de diversas formas, de acordo com o Portal de Classificação de Agrotóxicos, a classificação Carcinogênica IARC - Agência Internacional de Pesquisa do Câncer classifica se o agrotóxico é carcinogênico para os seres humanos. Com essa classificação, podemos observar na Tabela 2, que o Carbaril é carcinogênico, o carbofurano tem a probabilidade de ser carcinogênico, e o propoxur não é carcinogênico (ARIADNE, 2018).

De acordo com a classificação toxicológica da ANVISA (Agencia Nacional de Vigilância Sanitária), Tabela 2, para o carbaril e o propoxur recebe a classificação de Altamente tóxico (II), e o carbofurano é extremamente tóxico (I). Já o critério de classificação de agrotóxicos em função a Interferência Endócrina (IR), Tabela 2, para a vida humana, selvagem ou combinada, estabelece número 1 para agrotóxico que causam disrupção endócrina, 2 com potencial de causar a disrupção endócrina e 3 para quando não existe base cientifica (ARIADNE, 2018).

Ao avaliarmos os dados pode se aferir que o Carbaril e carbofurano para a vida selvagem possuem potencial de causar disrupção endócrina, e para a vida 
humana o carbaril possui disrupção endócrina e o carbofurano o potencial de disrupção, a mesma classificação nota se para a interferência endócrina combinada. Já para o propoxur não foi encontrado base cientifica para a vida humana, selvagem e combinada.

(ARIADNE, 2018).

Tabela 2. Classificação toxicológica quanto à carcinogenicidade, toxicidade e Interferência endócrina (IE).

\begin{tabular}{cccc} 
& Carbaril & Carbofurano & Propoxur \\
\hline $\begin{array}{c}\text { Classificação } \\
\text { IARC* }\end{array}$ & 1 & 3 & 4 \\
\hline $\begin{array}{c}\text { Classificação } \\
\text { ANVISA** }\end{array}$ & II & I & II \\
\hline $\begin{array}{c}\text { IR Vida } \\
\text { humana*** }\end{array}$ & 1 & 2 & - \\
\hline $\begin{array}{c}\text { IR Vida } \\
\text { Selvagem*** }\end{array}$ & 2 & 2 & - \\
\hline $\begin{array}{c}\text { IR } \\
\text { Combinada*** }\end{array}$ & 1 & 2 & - \\
\hline
\end{tabular}

Legenda:

* (1)- Carcinogênicos, (2A)- Probabilidade de carcinogênea, (2B)- Possibilidade de carcinogênea, (3)- não classificado, (4)- Não carcinogênicos.

** (I)- Extremamente Tóxico, (II)- Altamente Tóxico, (III)Mediamente Tóxico.

*** (1)- Disrupção endócrina, (2)- Potencial de causar a disrupção endócrina e (3)- Sem base científica.

Fonte: (ARIADNE, 2018).

Já que o Brasil é um dos líderes mundiais em consumo de agrotóxicos, o que torna o trabalho rural uma das ocupações mais perigosas da atualidade (FARIA et al., 2007). Inúmeras publicações cada vez mais têm apontado as intoxicações por agrotóxicos como grave problema de saúde pública (FARIA et al., 2004). Ainda, é preciso lembrar que, na agricultura, o local de trabalho é o ambiente, portanto, se contaminam o trabalhador, a produção e o ambiente. A exposição frequente a agrotóxicos pode causar danos à saúde do trabalhador rural e estas substâncias têm sido detectadas em amostras de sangue humano, leite materno e em resíduos de alimentos, o que aponta para a possibilidade de associação entre uso de agrotóxicos e ocorrência de doenças neurológicas, hepáticas, renais, respiratórias, imunológicas e endócrinas (MURAKAMI et al., 2017).

O fato é que a intensificação da agricultura tem prejudicado a biodiversidade, a exemplo da redução na disponibilidade e qualidade da água, do comprometimento da qualidade do ar e dos alimentos, e dos crescentes problemas fitossanitários resultantes do desequilíbrio ecológico causado pelo uso de agrotóxicos. No Brasil, estima se cerca de 15 milhões de pessoas que trabalham na agricultura estejam potencialmente expostas aos agrotóxicos, em quantidades bastante significativas. Dentre os casos, estima que anualmente ocorram de $150 \mathrm{mil} \mathrm{a} 200 \mathrm{mil}$ intoxicações agudas que não são propositais, com 3 mil a 4 mil óbitos (SILVA, 2009).

Considerando o aumento da produção agrícola do Tocantins, o crescimento do uso de pesticidas para esta produção, a importância do rio Formoso, para o desenvolvimento do estado e que as atividades antrópicas da região, como a agricultura, geram impacto no meio ambiente, fica evidenciado a importância deste trabalho. O monitoramento dos inseticidas da classe dos Carbamatos, apresentados na Tabela 1, além de fornecer um diagnóstico preliminar da situação da qualidade desse ecossistema aquático tão importante, pode servir de base para futuras pesquisas mais aprofundadas que ainda não existem no local estudado.

\section{MATERIAIS E MÉTODOS}

As amostras foram coletadas em 7 pontos do Rio Formoso, na região do Projeto Agrícola Formoso, em Formoso do Araguaia-TO, coordenadas apresentadas na Tabela 2. Foram realizadas 2 campanhas de coleta de amostras, em abril de 2018 (Campanha - C1), no período chuvoso, e em julho de 2018 (Campanha 2 - C2), período seco. 
Para as amostras de sedimento coletou-se aproximadamente $2 \mathrm{~kg}$ de sedimento da camada superficial e com distância de 5 a $10 \mathrm{~m}$ da margem do Rio em uma amostragem composta, com coletor Petersen modificado, de aço inox. Essa distância da margem escolhida para coleta variou em função da profundidade do rio, que se altera consideravelmente dependendo da época do ano. As amostras foram acondicionadas em frascos de polietileno descontaminados e acondicionadas em ambiente refrigerado com gelo até a chegada para preparação em laboratório (FILIZOLA et al., 2016).

As análises físico químicas do sedimento como $\mathrm{pH}\left(\mathrm{CaCl}_{2}\right)$, Acidez Potencial (H-Al), P, Carbono Orgânico Total, foram realizadas utilizando o método descrito por Donagema et al, (2011).

Para determinação dos carbamatos, as amostras de sedimento foram preparadas para análise por extração pelo método QuEChERS modificado. O procedimento do método QuEChERS modificado empregou como solvente de extração acetonitrila acidificada e para a etapa de partição os sais utilizados foram sulfato de magnésio e cloreto de sódio. Após a adição dos sais, os tubos foram agitados e centrifugados. A limpeza dos extratos foi realizada através da extração em fase sólida dispersiva (d-SPE, do inglês dispersive solid phase extraction). Empregou-se para a etapa de d-SPE sulfato de magnésio, e os sorventes octadecilsilano (C18) e amina primária secundária (PSA). Os extratos foram então agitados, centrifugados e filtrados. Antes da análise por UHPLC-MS/MS, as amostras foram diluídas cinco vezes em água ultrapura (PRESTES et al., 2009).

As análises foram realizadas por cromatografia líquida de ultra alta eficiência acoplada a espectrometria de massas em série (UHPLC-MS/MS, do inglês, ultra high performance liquid chromatography tandem mass spectrometry).

As amostras foram analisadas no sistema UHPLCMS/MS da Waters (EUA), equipado com: Cromatógrafo a líquido; detector MS do tipo triplo quadrupolo, modelo Xevo TQ; interface/fonte de ionização por eletro nebulização; gerador de nitrogênio peak; sistema controlador de solventes (sistema binário de bombas) para operação de gradientes a alta pressão; coluna analítica Acquity UPLC ${ }^{\circledR}$ BEH C18 $(50 \times 2,1$ $\mathrm{mm}, 1,7 \mu \mathrm{m}$ ) da Waters (EUA); sistema de aquisição de dados através do software MassLynx 4.1 (Waters, EUA). Monitoramento de reações selecionadas foram utilizadas para a quantificação e identificação dos analitos.

A fase móvel empregada foi (A) água:metanol (98:2, v/v) e (B) metanol, ambas contendo $5 \mathrm{mmol} \mathrm{L}^{-1} \mathrm{de}$ formiato de amônio e ácido fórmico 0,1\% (v/v); com vazão $0,225 \mathrm{~mL} \mathrm{~min}^{-1}$ e $10 \mu \mathrm{L}$ de volume de injeção. Utilizou-se modo de eluição gradiente [tempo (min), $\% \mathrm{~A}, \% \mathrm{~B}]:[0,95,5],[0.25,95,5],[7.75,5,95],[8.5,5$, 95], [8.51, 95, 5], [10, 95, 5], respectivamente (KEMMERICH, 2017).

\section{RESULTADOS E DISCUSSÃO}

Foram realizadas análises de caracterização físico química nas amostras de sedimento de fundo do Rio Formoso para melhor entendimento da dinâmica das substâncias estudadas neste meio.

Após essas análises verificou-se que quanto a textura do sedimento as características físico químicas apresentadas na Tabela 3, não apresentaram muita variação em função do período amostrado.

Os materiais erodidos (argila e matéria orgânica) do solo as margens de rios, carregam as moléculas de pesticidas e fertilizantes de áreas agrícolas para os mananciais. 
Tabela 3. Resultados obtidos das de textura, matéria orgânica e $\mathrm{pH}$ das amostras de sedimento das campanhas 1 e 2 .

\begin{tabular}{|c|c|c|c|c|c|}
\hline 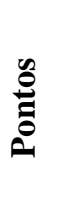 & 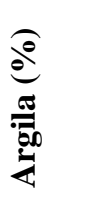 & 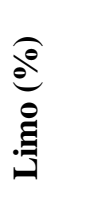 & 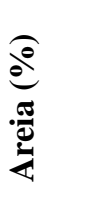 & 丞 & 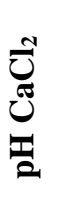 \\
\hline \multicolumn{6}{|l|}{$\mathrm{C1}$} \\
\hline 1 & 92,00 & 1,00 & 7,00 & 0,40 & 4,80 \\
\hline 2 & - & - & - & - & - \\
\hline 3 & 47,00 & 37,70 & 15,3 & 0,40 & 4,70 \\
\hline 4 & 7,00 & 1,00 & 92 & 0,20 & 5,60 \\
\hline 5 & 7,00 & 5,00 & 88 & 0,70 & 6,20 \\
\hline 6 & 7,00 & 1,00 & 92 & 0,40 & 5,90 \\
\hline 7 & 7,00 & 5,00 & 88 & 0,30 & 5,40 \\
\hline \multicolumn{6}{|c|}{$\mathrm{C2}$} \\
\hline 1 & 13,00 & 5,00 & 82 & 0,40 & 5,10 \\
\hline 2 & 10,00 & 6,80 & 88,2 & 0,40 & 6,20 \\
\hline 3 & 14,00 & 2,50 & 83,5 & 0,30 & 5,50 \\
\hline 4 & 11,00 & 2,50 & $86,5-$ & 0,40 & 5,50 \\
\hline 5 & 18,00 & 11,00 & 71 & 0,30 & 5,10 \\
\hline 6 & 7,00 & 1,00 & 92 & 0,10 & 5,30 \\
\hline 7 & 7,00 & 1,00 & 92 & 0,10 & 6,20 \\
\hline
\end{tabular}

Fonte: (AUTOR, 2018).

O Coeficiente de adsorção a matéria orgânica $\left(\mathrm{K}_{\mathrm{oc}}\right)$, indica a retenção do agrotóxico no solo, sendo utilizado para comparar a adsorção relativa do agrotóxico ao solo. Quando esse valor é alto, essa adsorção será também, logo, substâncias com alto $\mathrm{K}_{\mathrm{oc}}$ são pouco solúveis em água, podendo ser transportadas por partículas de sedimento, ao invés da água. Valores de $K_{o c}$ menor que 500 (baixa adsorção) indicam potencial considerável de perdas por lixiviação (AMARAL, 2011).

Como o Koc das substâncias determinam a capacidade de sorção dos pesticidas, capacidade essa relacionada com que o carbono orgânico do sedimento. Neste estudo observou-se que em sua maioria os sedimentos amostrados possuem baixo teor de matéria orgânica (exceção ponto 5 da campanha de coleta 1), apresentando em sua composição mais areia do que limo ou argila (com exceção do ponto 1 amostrado na campanha 1 período chuvoso), logo não ocorre a tendência das substâncias estudadas estarem sorvidas ao sedimento e sim dissolvida em água.

O ponto 5 da Campanha 1é o que apresenta maior concentração de matéria orgânica e os Pontos 6 e 7, coletados na Campanha 2 apresentam os valores mais baixos.

A característica de $\mathrm{pH}$ deste variando entre 5,10 a 6,2 , é outro fator que interfere na interação sedimento-molécula de pesticida.

Em períodos de muita chuva em pouco tempo é favorecido o escoamento superficial, erosão hídrica e degradação do solo. Novamente o ponto 1 e o ponto 3 da campanha 1 , se destacam dos demais em relação ao valor de $\mathrm{pH}$.

A constante de ionização $\left(\mathrm{K}_{\mathrm{a}} \mathrm{e} \mathrm{K}_{\mathrm{b}}\right)$, está relacionada com a capacidade do agrotóxico se ionizar, dentro de uma faixa de $\mathrm{pH}$ da solução do solo. Isso ocorre já que as diferentes formas do agrotóxico (molecular ou ionizada), apresentam comportamentos diferentes no solo. Em pH de 5 a 8, as moléculas dos agrotóxicos tendem a ficar carregadas negativamente e compostos aniônicos tendem a se lixiviar com mais facilidade que os catiônicos. Logo, quando o $\mathrm{pH}$ do meio é igual ao valor de $\mathrm{pk}_{\mathrm{a}}$ da espécie, observa-se uma ionização de $50 \%$. Quando temos $\mathrm{pH}$ maior que o valor de $\mathrm{pK}_{\mathrm{a}}$, predomina a fração aniônica e para $\mathrm{pH}$ menor que o Ka predomina a fração neutra (AMARAL, 2011).

Agrotóxicos ácidos são os que sua na sua forma molecular (neutra) podem doar prótons e formar ânions e agrotóxicos básicos a sua forma molecular (neutra) recebem prótons e se transformam em cátions.

No estado do Tocantins a predominância é do Latossolo. De forma geral os solos do estado, apresenta 
pH ácido, deficiência de nutrientes devido aos baixos teores de N, P, K, Ca, Mg, S, B, Cu, Mo, Zn e altos de $\mathrm{Al}$, que representa uma toxicidade deste elemento para plantas (MESQUITA, 2011; COBA, 2012). Ele possui também elevada capacidade de fixação de $\mathrm{P}$, com solos predominantemente das classes Cambisolos, Latosolos e Neossolos Quartzarenicos (MESQUITA, 2011).

Os valores de P e K do sedimento, confirmam a baixa fertilidade deste, conforme Tabela 4 .

Tabela 4. Resultados obtidos de teores de $\mathrm{P}, \mathrm{K}, \mathrm{Ca}, \mathrm{Al}, \mathrm{Mg}$ para determinação de índice de fertilidade.

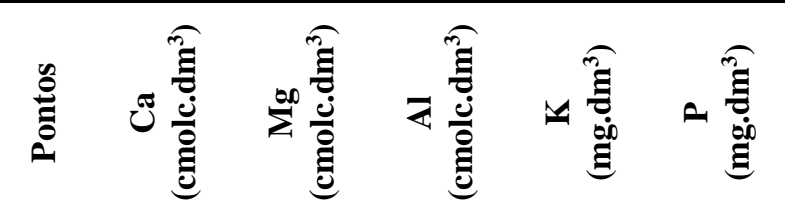

\begin{tabular}{llllll}
\hline $\mathbf{C 1}$ & & & & & \\
\hline $\mathbf{1}$ & 0,49 & 0,28 & 0,10 & 22,00 & 14,30 \\
\hline $\mathbf{2}$ & NA & NA & NA & NA & NA \\
\hline $\mathbf{3}$ & 2,42 & 1,69 & 0,20 & 29,00 & 5,00 \\
\hline $\mathbf{4}$ & 0,26 & 0,20 & 0,00 & 21,00 & 0,90 \\
\hline $\mathbf{5}$ & 0,57 & 0,46 & 0,10 & 20,0 & 16,00 \\
\hline $\mathbf{6}$ & 0,38 & 0,23 & 0,00 & 8,00 & 1,70 \\
\hline $\mathbf{7}$ & 0,56 & 0,40 & 0,10 & 15,00 & 11,80 \\
\hline $\mathbf{C 2}$ & & & & & \\
\hline $\mathbf{1}$ & 1,50 & 0,90 & 0,10 & 20,0 & 14,20 \\
\hline $\mathbf{2}$ & 1,80 & 1,50 & 0,00 & 21,00 & 9,20 \\
\hline $\mathbf{3}$ & 1,20 & 1,00 & 0,10 & 18,00 & 11,30 \\
\hline $\mathbf{4}$ & 1,60 & 1,00 & 0,1 & 18,00 & 9,70 \\
\hline $\mathbf{5}$ & 1,90 & 0,90 & 0,10 & 38,00 & 8,10 \\
\hline $\mathbf{6}$ & 0,30 & 0,10 & 0,10 & 15,00 & 6,50 \\
\hline $\mathbf{7}$ & 0,35 & 0,25 & 0,00 & 15,00 & 2,30 \\
\hline $\mathbf{n}$ & & & &
\end{tabular}

NA- não analisado. Fonte: (AUTOR, 2018).

Ao avaliarmos os resultados obtidos estão para os pesticidas estudados, verificou-se a não detecção dos princípios ativos. Como os resultados estão abaixo dos limites de detecção (LOD do inglês, limit of detection) e limites de quantificação (LOQ do inglês, limit of quantification) do método, em $\mu \mathrm{g} \cdot \mathrm{Kg}^{-1}$, considerou-se que os princípios ativos não estavam presentes nas amostras de sedimento. Estes valores limites estes são apresentados na Tabela 5, e variaram de acordo com a campanha analisada.

Tabela 5. Resultados obtidos das concentrações dos agrotóxicos carbaril, carbofurano e propoxur no sedimento das campanhas 1 e 2 .

\begin{tabular}{ccc}
\hline & LOD $\left(\mu \mathrm{g} . \mathrm{Kg}^{-1}\right)$ & LOQ $\left(\mu \mathrm{g} . \mathrm{Kg}^{-1}\right)$ \\
\hline Campanha 1 & & \\
\hline Carbaril & 0.003 & 0.008 \\
\hline Carbofurano & 0.003 & 0.008 \\
\hline Propoxur & 0.003 & 0.008 \\
\hline Campanha2 & & 0.008 \\
\hline Carbaril & 0.003 & 0.008 \\
\hline Carbofurano & 0.003 & 0.008 \\
\hline Propoxur & 0.003 &
\end{tabular}

Fonte: (AUTOR, 2018).

De acordo com os resultados obtidos de análise dos carbamatos no sedimento e analisando as características físico químicas como textura e matéria orgânica nas amostras, podemos entender essa não detecção dos princípios ativos. Outro fator que corrobora com essa não detecção é o conhecimento das propriedades dos agrotóxicos estudados apresentadas na Tabela 1. Ao analisar os valores de cada propriedade química de interesse do carbaril, carbofurano e propoxur, podemos observar que todos os pesticidas investigados apresentam rápida degradação no sedimento, e de média a alta mobilidade no solo, aliado ainda a uma média a alta solubilidade em água, com exceção do carbaril, conforme Tabela 6.

Vale ressaltar que embora não tenham sido encontrados no sedimento, isso não é indicativo de não estarem presentes no ecossistema aquático pois os princípios ativos apresentam potencial transporte para o corpo hídrico. A chuva, embora favoreça o transporte por escoamento superficial, também aumenta a vazão dos rios, ocasionando uma menor deposição no sedimento e uma maior diluição (CALHEIROS, et al., 
2018).

Tabela 6. Comportamento ambiental dos pesticidas estudados

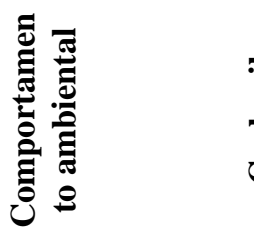

\begin{tabular}{llll}
\hline $\begin{array}{l}\text { Solubilidade } \\
\text { na água }\end{array}$ & Baixa & Média & Alta \\
\hline $\begin{array}{l}\text { Mobilidade } \\
\text { no solo }\end{array}$ & Média & Média & Alta \\
\hline $\begin{array}{l}\text { Persistência } \\
\text { no solo }\end{array}$ & Não & Não & Média \\
\hline $\begin{array}{l}\text { Hidrólise } \\
\text { na água }\end{array}$ & Não & Média & Alta \\
\hline $\begin{array}{l}\text { Degradação } \\
\text { no sedimento }\end{array}$ & Rápida & Rápida & Rápida \\
\hline
\end{tabular}

Fonte: Adaptado de PPDB, 2018.

A solubilidade em água $\left(\mathrm{S}_{\mathrm{W}}\right)$ é uma das características mais importantes, pois ela indica, a tendência do composto ser removido do solo. Essa remoção pode se dar por escoamento superficial ou lixiviação. $O$ valor de solubilidade nos mostra a probabilidade de uma agrotóxico atingir os corpos d'água (MILHOME, 2009).

Ter um valor alto de solubilidade não implica em maior mobilidade no solo, logo o que podemos observar é que além de ter alta solubilidade o agrotóxico também necessita ter alta mobilidade o que pode ser obtido com os valores de coeficiente de partição octanol/água (Kow) (MILHOME, 2009; AMARAL, 2011).

A detecção de pesticidas em ambientes naturais (não controlados), é difícil pois vários processos dinâmicos estão envolvidos nestes ambientes (diluição, dispersão, decomposição, hidrólise, fotólise) (CALHEIROS, et al., 2018).

Segundo USEPA (2012) parâmetros de qualidade de água como temperatura, $\mathrm{pH}$ e dureza podem aumentar a toxicidade de um pesticida e devem ser considerados em conjunto com a sua concentração quando quer se determinar o potencial de toxicidade deste para o ecossistema aquático.

Os princípios ativos, quando no ambiente, podem se degradar e formar outras substâncias, logo a não detecção de um princípio ativo não indica sua ausência no meio. Os produtos de degradação de um pesticida podem ter ou não toxicidade (USEPA, 2012).

Como exemplo da dificuldade de detecção de um pesticida temos o caso do carbaril, que ao sofrer hidrólise gera o 1-naltol, o seu principal subproduto de degradação. A fotólise aquosa é uma das vias de degradação e formação deste subproduto, ocorrendo na coluna superior da água, que permite maior passagem de luz. Em solos aeróbicos e sistemas aquáticos a degradação microbiológica ocorre rapidamente (USEPA, 2012).

Estudos sobre o 1-naftol são limitados. Esta substância é menos móvel que o carbaril e como pode estar presente no meio a partir de processos naturais e/ou antrópicos, sua presença não pode ser relacionada com o uso do carbaril, ficando evidenciado como os estudos e monitoramento de pesticidas é dificultoso e complexo (PPDB, 2018).

Segundo Martini et al. (2012), os modelos de predição do comportamento dos agrotóxicos no ambiente são muito úteis para se obter uma estimativa do risco de contaminação ambiental de algumas substâncias. Esses modelos auxiliam como uma análise preliminar na escolha dos agrotóxicos que devem ser monitorados já que vários estudos de monitoramento confirmam a tendência de comportamento de várias substâncias nos diferentes compartimentos ambientais. O uso de modelos de avaliação de risco, pode se prever o potencial de contaminação de águas superficiais e/ ou subterrâneas por determinado agrotóxico. Estes servem 
de base para indicar o comportamento ambiental da substância, podendo enquadrá-la com um risco maior ou menor de contaminação ambiental (MARQUES, 2005; MARTINI et al., 2012). Fazendo então, uma análise enquadrando e relacionando ao resultado negativo de agrotóxico no sedimento.

Ao avaliarmos o potencial de contaminação para águas subterrâneas dessas substâncias pelo índice GUS, podemos verificar que o carbaril possui um valor de GUS=1,157, logo, segundo este modelo, não sofre lixiviação, então é classificado como não contaminante (NC), ao passo que, o carbofurano GUS=3,493 e o propoxur GUS=3,73 apresentam valores superiores a 2,8, o que indica que provavelmente haverá lixiviação, ou seja, apresentam-se como potencial contaminante (PC) de aguas superficiais (HANSCH et al., 1995; SANTOS, 1999).

Ao averiguarmos as propriedades relacionadas aos critérios do método screening da EPA $\left(\mathrm{S}_{\mathrm{w}}, \mathrm{K}_{\mathrm{oc}}, \mathrm{K}_{\mathrm{H}}\right.$, $\mathrm{DT}_{50 \text { solo }}, \mathrm{DT}_{50 \text { água }}$ ), constatamos que para o Carbaril apenas o parâmetro $\mathrm{DT}_{50}$ água não se enquadra no método. No caso do carbofurano, $\mathrm{DT}_{50 \text { solo }}, \mathrm{DT}_{50}$ água, estão fora do critério, e para o propoxur $\mathrm{DT}_{50 \text { solo }}, \mathrm{e}_{50}$ água, estão fora dos critérios do método.

Fica demonstrado, então, que segundo o método EPA são considerados contaminantes em potencial de águas subterrâneas, os agrotóxicos Carbaril, carbofurano e propoxur.

No entanto, o Carbaril, segundo GUS, não sofre lixiviação. Para o carbofurano e propoxur, os dois modelos mostram concordância, sendo assim classificados com alto potencial de contaminação de águas subterrâneas.

Com a análise dos 2 métodos, pode-se verificar que o Carbaril é um contaminante intermediário, já o carbofurano e o propoxur são classificados como contaminantes potencial de águas subterrâneas, como mostrados na Tabela 7.

Tabela 7. Resultados obtidos com o método screening da EPA e índice GUS, para avaliação do potencial de contaminação de águas subterrâneas, dos agrotóxicos Carbaril, Carbofurano e Propoxur.

\begin{tabular}{cccc}
\hline Agrotóxico & Índice GUS & Método da EPA & $\begin{array}{c}\text { Risco de contaminação para } \\
\text { água superficial }\end{array}$ \\
\hline Carbaril & Não sofre Lixiviação & Contaminante Potencial & Intermediário Cont. Potencial \\
\hline Carbofurano & Sofre Lixiviação & Contaminante Potencial & Contaminante Potencial \\
\hline Propoxur & Sofre Lixiviação & Contaminante Potencial & Contaminante Potencial
\end{tabular}

Fonte: (Adaptado de MARTINI, 2012).

Quando analisamos as propriedades para o método GOSS, percebemos que o propoxur apresenta baixo potencial de contaminação associado ao transporte de sedimento, enquanto o potencial de transporte dissolvido em água é alto, representando um alto risco de contaminação para águas superficiais (Tabela 8). Os demais pesticidas possuem baixo potencial de transporte associado ao sedimento e médio potencial de contaminação dissolvido em água.
Tabela 8. Resultados obtidos com o método GOSS, para avaliação do potencial de contaminação de águas superficiais, dos pesticidas estudados.

\begin{tabular}{ccc}
\hline & $\begin{array}{c}\text { Potencial de } \\
\text { transporte associado } \\
\text { ao sedimento }\end{array}$ & $\begin{array}{c}\text { Potencial de } \\
\text { transporte } \\
\text { dissolvido em água }\end{array}$ \\
\hline Carbaril & Baixo & Médio \\
\hline Carbofurano & Baixo & Médio \\
\hline Propoxur & Baixo & Alto \\
\hline
\end{tabular}

Fonte: Adaptado de MARTINI et al., 2012; LIMA, 2017). 
Embora não se tenha conseguido neste trabalho detectar os princípios ativos investigados, aanálise das características físico químicas do sedimento de fundo do Rio Formoso, das características físico químicas dos princípios ativos estudados e investigação dos modelos de potencial de contaminação, podemos sugerir que se presentes no meio ambiente da região estudada, essas substâncias tenderão a estar dissolvidas na água do rio.

\section{CONCLUSÃO}

Os resultados obtidos com este estudo, mostraram a necessidade de um estudo mais completo de determinação dos princípios ativos investigados em diferentes compartimentos ambientais do Rio Formoso, já que não foram detectados no sedimento de fundo deste Rio.

Estudos de monitoramento mais aprofundado de pesticidas, na água, solo e em organismos aquáticos deste rio, faz-se necessária, já que a região tem um forte crescimento agrícola e consequentemente um aumento no uso dessas substâncias para garantir essa o aumento da produção de grãos.

\section{AGRADECIMENTO}

Universidade Federal do Tocantins e Programa de Pós-Graduação em Biodiversidade e Biotecnologia - BIONORTE; e Laboratório de Química e Biocombustíveis Ambientais (LAPEQUFT). O presente trabalho foi realizado com o apoio da Coordenação de Aperfeiçoamento de Pessoal de Nível Superior Brasil - (CAPES) - Código de Financiamento [001].

Todos os autores declararam não haver qualquer potencial conflito de interesses referente a este artigo.

\section{REFERÊNCIAS}

ADAPEC, Agência de Defesa Agropecuária do Estado do Tocantins. Cadastramento e Inspeção do Comércio de Agrotóxicos, 2008. Disponível em: http://adapec.to.gov.br/cadastramento-de-empresasprodutoras-e-produtos-agrotoxicos/ Acesso em 06/03/2018.

AMARAL, A. B., Avaliação de mananciais subterrâneos e superficiais da bacia do Córrego Sossego considerando o uso para abastecimento doméstico e irrigação - contaminação por agrotóxico. Dissertação de Mestrado em Engenharia Ambiental- Centro Tecnológico, Universidade Federal do Espírito Santo, Vitória, 2011.

ANDRADE, A. S., QUEIROZ, V. T., LIMA, D. T., DRUMOND, L. C. Análise de risco de contaminação de águas superficiais e subterrâneas por pesticidas em municípios do Alto Paranaiba-Mg. Revista Química nova, v.34, n. 7, Abril, p. 1129-1135, 2011.

ANVISA. Agência Nacional de Vigilância Sanitária. Programa de Análise de Resíduos de Agrotóxicos em Alimentos (PARA). Relatório de Atividades de 2011 e 2012. Brasília; 2013. Disponível em: < http://bibspi.planejamento.gov.br/handle/iditem/448> . Acesso em 05/03/2018.

ARIADNE. Sistema de informação sobre agrotóxico. Comportamento ambiental. Disponível em: < http://www.fsp.usp.br/nra/ariadne/comportamentoambiental.php>. Acesso em 07/08/2019.

BOMBARDI, L. M. Geografia do Uso de Agrotóxicos no Brasil e Conexões com a União Europeia / Larissa Mies Bombardi. - São Paulo: FFLCH - USP. 296 p., 2017 ISBN:978-85-7506-31015.

CALDAS, E. D.; SOUZA, L. C. K. Avaliação de risco crônico da ingestão de resíduos de pesticidas na dieta brasileira. Revista Saúde Pública. São Paulo, v.34, n. 5, p.529-537, 2000.

CALHEIROS, D. F.; PIGNATI, W. A., PINHO, A. P.; SOUZA E LIMA, F. A. N.; SANTOS, J.; PINHO, J. S.; ROSA, E. R., Relatório Técnico Projeto: Promoção da Agroecologia e Avaliação da Contaminação por Agrotóxicos em Áreas de Proteção Ambiental na Bacia do Alto Paraguai APA Estadual Nascentes do Rio Paraguai-Ministério da Educação, Universidade Federal de Mato Grosso Instituto de Saúde Coletiva/Departamento de Saúde Coletiva Núcleo de Estudos Ambientais e Saúde do Trabalhador, $2018 . \quad$ Disponível em: http://ecoa.org.br/wpcontent/uploads/2018/05/988025221b5fb8dd47b5033 4964de19e.pdf Acesso 16/04/2019 
COBA, M. S. M. Diversidade de bactérias cultiváveis no solo do Cerrado Brasileiro. Dissertação Mestrado em Microbiologia Agrícola, Universidade Federal de Lavras, Lavras., 2012.

DELLAMATRICE, P. M.; MONTEIRO, R. T. R. Principais aspectos da poluição de rios brasileiros por pesticidas. R. Bras. Eng. Agríc. Ambiental, v.18, n.12, p.1296-1301, 2014.

DONAGEMA, G. K. Manual de métodos de análise de solos / organizadores, Guilherme Kangussú Donagema... [et al.]. — Dados eletrônicos. — Rio de Janeiro : Embrapa Solos, 2011.

FARIA, N. M. X., FACCHINI, L. A., FASSA, A. C. G., TOMASI, E. Trabalho rural e intoxicações por agrotóxicos. Caderno de Saúde Pública, Rio de Janeiro, v.20, n.5, p.1298-1308, 2004.

FARIA, N. M. X.; FASSA, A. C. G.; FACCHINI, L. A. Intoxicação por agrotóxicos no Brasil: os sistemas oficiais de informação e desafios para realização de estudos epidemiológicos. Ciência \& Saúde Coletiva, v.12, n.1, p.25-38, 2007.

FERRACINI, V.L.; PESSOA, M.C.Y.P.; SILVA, A.S.; SPADOTTO, C.A. Análise de risco de contaminação das águas subterrâneas e superficiais da região de Petrolina (PE) e Juazeiro (BA). Pesticidas: Revista de Eco toxicologia e Meio Ambiente, v. 11, p. 1-16, 2011.

FILIZOLA, E. F.; GOMES, M. A. F. SOUZA, M.D., Manual de procedimentos de coleta de amostras em áreas agrícolas para análise da qualidade ambiental: solo, água e sedimentos. Jaguariúna: Embrapa Meio Ambiente, 2016.

GAMA, A. F.; OLIVEIRA, A. H. B.; CAVALCANTE, R. M. Inventário de agrotóxicos e risco de contaminação química dos recursos hídricos no semiárido cearense. Quím. Nova. vol.36, n.3, pp.462-467. 2013.

GOSS, D.W. Screening procedure for soils and pesticides for potential water quality impacts. Weed Technology, v. 6, n. 3, p. 701-708, 1992.

GUSTAFSON, D.I. Groundwater Ubiquity Score: a simple method for assessing pesticide leachability. Environmental Toxicology and Chemistry, v. 8, n. 4, p. 339-357.1989.

HANSCH, C.; LEO, A.; HOEKMAN, D.. Exploring QSAR - Hydrophobic, Electronic, and Steric
Constants. Washington, DC: American Chemical Society., p. 88, 1995.

KEMMERICH, M. Resíduos de agrotóxicos em ameixa, maçã, pera e pêssego: desenvolvimento de métodos de análise e monitoramento. Tese doutorado em Química, Universidade Federal de Santa Maria, Santa Maria, 2017

KIDD, H., JAMES, D. R. The Agrochemicals Handbook, Third Edition. Royal Society of Chemistry Information Services, Cambridge, UK, 311, 1991 (as updated).

LIMA, J. A. M. C. Ocorrência de agrotóxicos em águas rurais e bioacumlados em biofilmes epilíticos do Rio Grande d Sul. Tese de Doutorado. Curso de Pós Graduação, Universidade Federal de Santa Maria, Santa Maria, 2017.

MARQUES, M.N. Avaliação do impacto de agrotóxicos em áreas de proteção ambiental, pertencentes à bacia hidrográfica do Rio Ribeira de Iguape, São Paulo: uma contribuição à análise crítica da legislação sobre o padrão de potabilidade. Tese de Doutorado. Universidade de São Paulo, São Paulo, 2005.

MARQUES, M.N. Avaliação do impacto de agrotóxicos em áreas de proteção ambiental, pertencentes à bacia hidrográfica do Rio Ribeira de Iguape, São Paulo: uma contribuição à análise crítica da legislação sobre o padrão de potabilidade. 2005. Tese de Doutorado. Universidade de São Paulo, São Paulo, 2005.

MARTINI, L. F. D.; CALDAS, S. S.; BOLZAN, C. M.; BUNDT, A. C.; PRIMEL, E. G.; AVILA, L. A. Risco de contaminação das águas de superfície e subterrâneas por agrotóxicos recomendados para a cultura do arroz irrigado. Ciencia Rural, v.42, n.10, p.1715-1721. 2012.

MESQUITA, V. A. Caracterização da diversidade microbiológica de solos do Cerrado de Minas Gerais por eletroforese em gel de gradientes desnaturante (DGGE). Dissertação de Mestrado em Microbiologia Agrícola. Universidade Federal de Lavras, Lavras, 2011.

MILHOME, M. A. L. Avaliação do potencial de contaminação de águas superficiais e subterrâneas por pesticidas aplicados na agricultura do Baixo Jaguaribe, CE. Eng. Sanit. Ambiental v. 14, n. 3, p.363-372, set. 2009.

MURAKAMI, Y., PINTO, N. F., ALBUQUERQUE, G. S. C., PERNA, P. O., LACERDA, A. Intoxicação 
crônica por agrotóxicos em fumicultores. Saúde Debate, Rio de Janeiro, v. 41, n. 113, p. 563-576, 2017.

PRIMEL，E.G.; ZANELLA，R.; KURZ，M.H.S.; GONÇALVES, F.F.; MACHADO, S.O.; MARCHEZAN, E. (2005) Poluição das águas por herbicidas utilizados no cultivo do arroz irrigado na região central do estado do Rio Grande do Sul, Brasil: predição teórica e monitoramento. Química Nova, v. 28, n. 4, p. 605-609. n.10, pp.1715-1721, 2012.

POSSAVATZ, J. Pesticide residue in bottom sediment in the Cuiabá River watershed, Mato Grosso, Brazil. Rev. Ambient. Água[online]. v.9., n.1, p.83-96., 2014 ISSN 1980-993X. http://dx.doi.org/10.4136/ambiagua. 1263.

PPDB -THE PESTICIDE PROPERTIES DATABASE Other product constituents Insecticides Herbicides-Fungicides. University of Hertfordshire, funded by UK National Sources and the EU-funded FOOTPRINT project. 2018. Disponível em: https://sitem.herts.ac.

uk/aeru/ppdb/en/Reports/118.htm\#trans>. Acesso em 08/04/2019.

PRESTES, O.D.; FRIGGI, C.A.; ADAIME, M. B.; ZANELLA, R. QuEChERS - um método moderno de preparo de amostra para determinação multirresíduo de pesticidas em alimentos por métodos cromatográficos acoplados à espectrometria de massas. Química Nova, Vol. 32, No. 6, 1620-1634, 2009.

PUBCHEM. Centro Nacional de Informações sobre Biotecnologia. Banco de Dados Composto PubChem; CID $=2566$. Disponível em: $<$ https://pubchem.ncbi.nlm.nih.gov>. Acesso em 10/08/2018.

REBELO, R.; CALDAS, E. D. Avaliação de risco ambiental de ambientes aquáticos afetados pelo uso de agrotóxicos. Quím. Nova. vol.37, n.7, pp.1199-1208, 2014.

RIBEIRO, D.H.B.; VIEIRA, E. Avaliação do potencial de impacto dos agrotóxicos no meio ambiente. 2010. Artigo em Hipertexto. Disponível em:

<http://www.infobibos.com/Artigos/2010_2/agrotoxi cos/index.htm>. Acesso em:03/03/2018.

SANTOS, T. C. R. Estudo da degradação do herbicida propanil em campos de cultivo de arroz. Tese de doutorado. Universidade Estadual Paulista, Instituto de Química, São Paulo, 1999.

SILVA, M. N., Multiresidual Method Development to analysis of pesticides in honey. Using liquid chromatography with spectrophotometric detector with arrangement of diodes. Dissertação de Mestrado em Química. UFMA, São Luíz, 2009.

SOARES, D. F.; FARIA, A. M.; ROSA, A. H. Análise de risco de contaminação de águas subterrâneas por resíduos de agrotóxicos no município de Campo Novo do Parecis (MT), Brasil. Eng. Sanit. Ambient. [online]. 2017, vol.22, n.2, pp.277-284. Epub Oct 27, 2016.

SOARES, E. M., LIMA, S. M., PEDRO, L. P., LUCENA, A. R. F. P., SOUZA, J. F. S. Plano estadual de vigilância em saúde de populações expostas a agrotóxicos do estado do Tocantins. Secretaria de Estado da Saúde. Palmas, 2013. Disponível em: http://portalarquivos.saude.gov.br/images/pdf/2015/ju ho/08/PlanoTO.pdf Acesso em 10/08/2018.

SPADOTTO, C. A.; Abordagem Interdisciplinar Na Avaliação Ambiental De Agrotóxicos. Núcleo de Pesquisa Interdisciplinar, São Manuel, 2006. 9p.

USEPA - United States Environmental Protection Agency- Aquatic life ambient e water quality criteria for carbaryl- EPA-829-R-007 April, 2012.

WAUCHOPE, R. D.; BUTTLER, T. M.; HORNSBY, A. G.; AUGUSTIJN-BECKERS, P. W. M.; BURT, J. $\mathrm{P}$. The SCS/ARS/CES pesticides properties database for environmental decision-making. Reviews of Environmental Contamination and Toxicology, v. 123, p. 1-35, 1992. 